\title{
Cenário epidemiológico da tuberculose no município de Sobral (CE) entre os anos de 2013 e 2017
}

\author{
Epidemiological scenario of tuberculosis in the city of Sobral (CE) between the years of \\ 2013 and 2017 \\ Cenario epidemiológico de la tuberculosis en el municipio de Sobral (CE) entre los años \\ de 2013 y 2017
}

Aline Costa Souza ${ }^{1 *}$, Feliphy Rodrigues Custódio ${ }^{1}$, Olindina Ferreira Melo.

\begin{abstract}
RESUMO
Objetivo: Analisar a condição epidemiológica da tuberculose no município de Sobral (CE) entre os anos de 2013 e 2017. Métodos: O estudo está sob o aspecto epidemiológico quantitativo mediante o levantamento de dados de domínio público e de abrangência nacional, alimentados por meio da notificação compulsória de doenças e agravos. A pesquisa foi realizada com base nos elementos epidemiológicos da cidade de Sobral, região Norte do Ceará, com dados referentes ao período de 2013 a 2017. Resultados: Foram notificados 993 casos de tuberculose no município, sendo tais acometidos adultos jovens, entre 20 e 59 anos, somando 721 casos. Os indivíduos do sexo masculino foram mais prevalentes em relação ao sexo feminino. A grade maioria dos episódios notificados constituiu de casos novos em todos os anos estudados. Os casos de tuberculose pulmonar constituíram $84 \%$ e a maior parcela dos casos notificados (54\%) era positiva para o bacilo na primeira baciloscopia. A cura constituiu maior percentual entre os encerramentos dos casos. Conclusão: Os resultados explanam a situação epidemiológica de tuberculose em Sobral (CE) e apontam para um fortalecimento nas estratégias dos serviços de saúde, principalmente relacionado ao diagnóstico precoce dos casos, com a finalidade de combater a doença no município.
\end{abstract}

Palavras-chave: Tuberculose, Epidemiologia, Saúde Pública.

\begin{abstract}
Objective: To analyze the epidemiological condition of tuberculosis in the municipality of Sobral (CE) between the years 2013 and 2017. Methods: The study is based on the quantitative epidemiological aspect through the collection of data from a public body and national scope, fed by the environment of the compulsory obligation of diseases and injuries. The research was carried out based on the epidemiological elements of the city of Sobral, northern region of Ceará, with data for the period from 2013 to 2017. Results: 991 cases of tuberculosis were reported in the city, and young adults were affected between 20 and 59721 cases. Males were more prevalent than females. Most of the reported episodes constitute the new cases in all the years studied. The cases of pulmonary tuberculosis constituted $84 \%$ and the highest instance of reported cases (54\%) was positive for the bacillus in the first bacilloscopy. The constitution of the highest percentage of clinical cases. Conclusion: The results explain the epidemiological situation of tuberculosis in Sobral (CE) and point to a strengthening in the relations of the health services, indicating mainly the antecedents of the cases, with a purpose to fight a disease in the municipality.
\end{abstract}

Key words: Tuberculosis, Epidemiology, Public Health.

${ }^{1}$ Centro Universitário INTA (UNINTA), Sobral-Ceará. *E-mail: aline.c.souza2013@gmail.com SUBMETIDO EM: 2/2019 ACEITO EM: 3/2019 PUBLICADO EM: 5/2019 


\section{RESUMEN}

Objetivo: Analizar la condición epidemiológica de la tuberculosis en el municipio de Sobral (CE) entre los años 2013 y 2017. Métodos: El estudio está bajo el aspecto epidemiológico cuantitativo a través del levantamiento de datos de una instancia pública y de alcance nacional, alimentados por el medio de la obligación obligatoria de enfermedades y agravios. La investigación fue realizada con base en los elementos epidemiológicos de la ciudad de Sobral, región Norte de Ceará, con datos relativos al período de 2013 a 2017.

Resultados: Se notificaron 991 casos de tuberculosis en el municipio, siendo acosados adultos jóvenes, entre 20 y 59 años, sumando 721 casos. Los individuos del sexo masculino fueron más prevalentes en relación al sexo femenino. La mayoría de los episodios notificados constituyen los nuevos casos en todos los años estudiados. Los casos de tuberculosis pulmonar constituyeron el $84 \%$ y la mayor instancia de casos notificados (54\%) fue positiva para el bacilo en la primera baciloscopia. La constitución del mayor porcentaje de casos clínicos de casos. Conclusión: Los resultados explican la situación epidemiológica de tuberculosis en Sobral (CE) y apuntan a un fortalecimiento en las relaciones de los servicios de salud, indicando principalmente los antecedentes de los casos, con la finalidad de combatir una enfermedad en el municipio.

Palabras clave: Tuberculosis, Epidemiología, Salud Pública.

\section{INTRODUÇÃO}

A tuberculose (TB) é uma enfermidade infectocontagiosa causada pelo Mycobacterium tuberculosis, também conhecido como Bacilo de Koch (BK). É um bacilo álcool-ácido resistente (BAAR), o qual faz parte de um complexo constituído de várias espécies de bactérias, cujo principal reservatório é o homem. A TB é transmitida entre pessoas por meio de gotículas contendo o bacilo, disseminadas pela fala, espirro e tosse (BRASIL, 2010).

Apesar de ser uma doença com diagnóstico simples, tratamento e vacina disponível na rede pública de saúde, ainda consiste em um problema pertinente entre a população, atingindo todas as faixas etárias e agravando-se em decorrência de fatores sociais e econômicos menos favoráveis, onde os principais pontos que dificultam o controle estão intimamente ligados à prevenção, diagnóstico, tratamento, qualidade dos serviços de saúde e aspectos relacionados aos pacientes (CECILIO HPM, et al., 2013; CECILIO HPM, et al., 2017).

É importante destacar os principais fatores de risco relacionados à transmissão da tuberculose, como por exemplo, o diagnóstico recente de tuberculose em pessoas da família ou de convívio próximos, a infecção pelo vírus da imunodeficiência humana, a presença de enfermidades associadas como diabetes, câncer ou doenças pulmonares crônicas, além do excesso no consumo de álcool e/ou drogas ilícitas e a habitação dos indivíduos, sendo que o risco de transmissão da doença aumenta em áreas comunitárias e entre os moradores de ruas (ALCOBIA CS e COSTA SAN, 2016).

O diagnóstico da tuberculose baseia-se em dois aspectos fundamentais, com a observância dos sinais e sintomas clínicos e dos exames laboratoriais bacteriológicos. Dentre estes métodos diagnósticos estão a baciloscopia direta do escarro, cultura de escarro, exame radiológico, tomografia de tórax, broncoscopia, prova tuberculínica, exames histológicos, citológicos, bioquímicos, sorológicos e de biologia molecular (BRASIL, 2010).

Além dos métodos clássicos citados, em 2014, houve a implantação do teste rápido molecular para tuberculose (TRM-TB), desenvolvido para identificar ao mesmo tempo o Mycobacterium tuberculosis e a resistência à rifampicina (RIF) em aproximadamente 2 horas. O método emprega a tecnologia de reação em cadeia da polimerase (PCR) em tempo real para extração, amplificação e detecção do DNA do microrganismo e triagem de cepas resistentes à rifampicina (BRASIL, 2015). As vantagens deste método incluem a alta sensibilidade, maior que a da baciloscopia, e a alta especificidade para o Mycobacterium tuberculosis, além da detecção da resistência à rifampicina, o que promove melhoria no diagnóstico e no tratamento da doença (BRASIL, 2016c). 
Na Atenção Primaria a Saúde (APS), as equipes de saúde buscaram nos últimos anos implantar estratégias para o controle da tuberculose incluindo em suas ações a busca ativa de pacientes sintomáticos, pedidos de exames para diagnóstico, efetivação de registro das informações acerca da doença, bem como a inserção do tratamento supervisionado e da família no tratamento dos doentes com o intuito de aplicar medidas participativas, coletivas e integrais para garantir uma maior abrangência do controle da TB (CECILIO HPM, et al., 2015).

A Organização Mundial de Saúde (OMS) reconheceu a tuberculose, em 1993, como uma emergência global, a qual foi inserida nas políticas públicas internacionais com o intuito de reduzir e parar o coeficiente de incidência da doença até 2015. A partir desta iniciativa foi observada uma melhoria no cenário mundial da tuberculose, no qual 43 milhões de pessoas foram salvas, entre 2000 e 2014, por meio do diagnóstico eficaz e tratamento efetivo da doença (OMS, 2015).

Apesar disso, apresenta um coeficiente de mortalidade ainda alarmante nos dias atuais, com 2,2 por 100 mil habitantes em 2015, e configura-se como um problema de saúde pública no Brasil. Segundo a OMS, em 2014 um milhão de pessoas no mundo morreram em decorrência da tuberculose e no Brasil o número de mortes chegou a 4.400 (BRASIL, 2016b). Em relação à nova classificação da OMS (2016-2020), o Brasil atualmente ocupa a $20^{\underline{a}}$ posição na lista dos 30 países com alta carga de TB (BRASIL, 2016d).

O Ceará em 2014 apresentou um coeficiente de incidência de tuberculose de 35,4 casos por 100 mil habitantes, maior que o nacional (29,0/100mil hab.), ocupando a 9a posição no ranking dos estados com maior coeficiente de incidência da doença e $8^{\underline{a}}$ posição no coeficiente de mortalidade por tuberculose (BRASIL, 2016b; BRASIL, 2016d).

Ainda, foi observada uma redução da incidência dos casos de tuberculose de 39,4/100 mil habitantes em 2013 para 38,3/100 mil habitantes em 2017, o que representa uma diminuição de 1,1\%. É importante salientar que a meta estabelecida pelo Ministério da Saúde é de menos de 10 casos para cada 100 mil habitantes. Os óbitos por tuberculose, em 2016, superaram os causados pela Síndrome da Imunodeficiência Adquirida (AIDS), ressaltando que a TB é uma doença evitável, indicando uma fragilidade dos serviços de atenção básica em relação à assistência ao paciente (CEARÁ, 2018).

Diante das considerações expostas, o presente artigo tem por objetivo avaliar o perfil epidemiológico da tuberculose no município de Sobral no estado do Ceará referente ao período de 2013 a 2017. Para tanto, foram observados o quantitativo dos casos notificados quanto à estratificação conforme faixa etária e sexo, a forma clínica mais acometida na população notificada, os resultados obtidos nos exames de baciloscopia direta de escarro e, ainda, averiguar a situação de encerramento do caso.

\section{MÉTODOS}

O estudo está sob o aspecto epidemiológico quantitativo mediante o levantamento de dados de domínio público e de abrangência nacional, obtidos na página eletrônica do Departamento de Informática do Sistema Único de Saúde (DATASUS), alimentados por meio da notificação compulsória de doenças e agravos pertencentes à lista nacional do SINAN (Sistema de Informação de Agravos de Notificação). A pesquisa foi realizada com base nos elementos epidemiológicos da cidade de Sobral, região Norte do Estado do Ceará, com dados referentes ao período de 2013 a 2017.

Os critérios de inclusão corresponderam aos casos confirmados de indivíduos infectados pela bactéria Mycobacterium tuberculosis na cidade de Sobral (CE) notificados à Secretaria de Saúde do Estado de Ceará no período de 2013 a 2017. Como critérios de exclusão foram considerados os casos notificados até dezembro de 2012 e após dezembro de 2017, assim como casos inconclusivos ou ignorados para a tuberculose na cidade de Sobral (CE), além dos casos de pessoas que residem em outros municípios do Estado do Ceará.

As variáveis estudadas incluíram o quantitativo de casos de tuberculose notificados na cidade de Sobral, variáveis sociais como faixa etária e sexo, resultado da primeira baciloscopia de escarro, forma clínica da 
doença e situação de encerramento (SINAN, 2018). As tabelas foram geradas pelo sistema DATASUS e exportadas para o Microsoft Office Excel $2010^{\circledR}$. Os dados coletados foram expostos na forma de tabelas e gráficos e, em seguida, comentados e relacionados com o embasamento literário.

A coleta de dados secundários, por intermédio de informações de saúde, epidemiológicas e morbidades originárias do DATASUS, é de domínio público, de forma que a presente pesquisa não careceu de aprovação em comitê de ética de pesquisa ou de comissão científica local, conforme a Resolução o 510 de 2016 do Conselho Nacional de Saúde. Portanto, a realização deste estudo implicou nos cumprimentos dos quesitos de autonomia, beneficência, não maleficência, justiça e equidade.

\section{RESULTADOS}

A pesquisa realizada mostrou que entre os anos de 2013 e 2017 foram notificados 993 casos de tuberculose no município de Sobral (CE). Os indivíduos do sexo masculino foram mais prevalentes em relação ao sexo feminino totalizando $67 \%$ dos casos. O ano de 2014 apresentou-se com o maior número de notificações, contabilizando 217 casos, enquanto que em 2016 apenas 182 casos foram notificados, apresentando-se com o menor número de casos notificados (Gráfico 1).

Gráfico 1 - Casos de tuberculose notificados entre os anos de 2013 e 2017 na cidade de Sobral (CE).

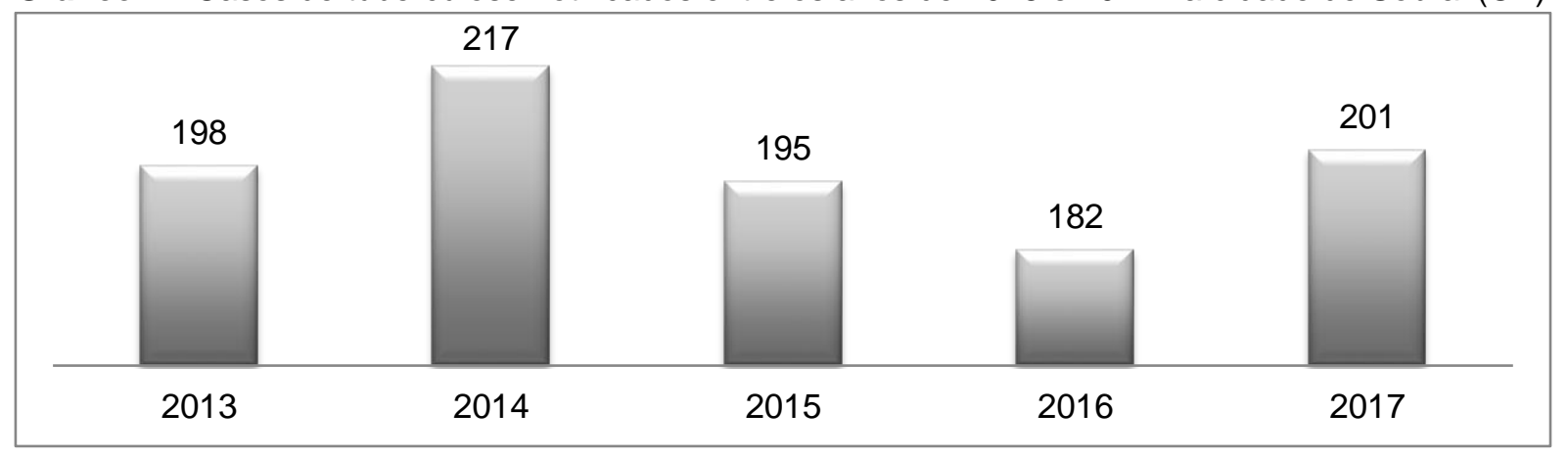

Fonte: Dados da Pesquisa. Ministério da Saúde/SVS - Sistema de Informação de Agravos de Notificação SINAN Net.

Quando se observam os casos notificados em relação à faixa etária, nota-se que adultos jovens, entre 20 e 59 anos, foram os mais acometidos pela tuberculose em todos os anos avaliados, somando 721 casos entre 2013 e 2017. Indivíduos entre 15 e 19 anos foram o segundo grupo mais atingido pela doença, totalizando 82 casos notificados no período estudado (Tabela 1).

Tabela 1 - Número de casos de tuberculose, conforme faixa etária, notificados entre os anos de 2013 e 2017 na cidade de Sobral (CE).

\begin{tabular}{ccccccccccccc}
\hline Faixa & $<1$ & $\mathbf{0 1} \mathbf{a}$ & $\mathbf{0 5} \mathbf{a}$ & $\mathbf{1 0} \mathbf{a}$ & $\mathbf{1 5} \mathbf{a}$ & $\mathbf{2 0 ~ A}$ & $\mathbf{4 0 ~ A}$ & $\mathbf{6 0 ~ A}$ & $\mathbf{6 5 ~ A}$ & $\mathbf{7 0 ~ A}$ & $\mathbf{8 0}$ & TOTAL \\
Etária & Ano & $\mathbf{0 4}$ & $\mathbf{0 9}$ & $\mathbf{1 4}$ & $\mathbf{1 9}$ & $\mathbf{3 9}$ & $\mathbf{5 9}$ & $\mathbf{6 4}$ & $\mathbf{6 9}$ & $\mathbf{7 9}$ & $\mathbf{e}+$ & + \\
\hline $\mathbf{2 0 1 3}$ & - & - & - & 6 & 16 & 103 & 50 & 10 & 5 & 6 & 2 & $\mathbf{1 9 8}$ \\
$\mathbf{2 0 1 4}$ & 1 & 2 & 2 & 6 & 16 & 96 & 63 & 4 & 8 & 14 & 5 & $\mathbf{2 1 7}$ \\
$\mathbf{2 0 1 5}$ & - & - & 2 & 5 & 15 & 78 & 60 & 8 & 7 & 15 & 5 & $\mathbf{1 9 5}$ \\
$\mathbf{2 0 1 6}$ & 1 & - & 1 & 2 & 22 & 77 & 51 & 10 & 6 & 7 & 5 & $\mathbf{1 8 2}$ \\
$\mathbf{2 0 1 7}$ & 1 & 2 & 1 & 7 & 13 & 89 & 54 & 8 & 14 & 8 & 4 & $\mathbf{2 0 1}$ \\
\hline TOTAL & $\mathbf{3}$ & $\mathbf{4}$ & $\mathbf{6}$ & $\mathbf{2 6}$ & $\mathbf{8 2}$ & $\mathbf{4 4 3}$ & $\mathbf{2 7 8}$ & $\mathbf{4 0}$ & $\mathbf{4 0}$ & $\mathbf{5 0}$ & $\mathbf{2 1}$ & $\mathbf{9 9 3}$ \\
\hline
\end{tabular}

Fonte: Dados da pesquisa. Ministério da Saúde/SVS - Sistema de Informação de Agravos de Notificação Sinan Net. 
A maioria dos episódios notificados constituiu de casos novos em todos os anos estudados, com um total de 815 novas notificações. Os casos de reingresso após abandono do tratamento mostraram-se como segundo maior registro de tipo de entrada, constituindo $8 \%$ dos eventos notificados. Em adição, observaramse 3 casos de notificação da tuberculose no pós-óbito, ou seja, casos registrados após a morte do paciente, em decorrência da realização de investigação epidemiológica (Gráfico 2).

Gráfico 2 - Casos de tuberculose, conforme tipo de entrada, notificados entre os anos de 2013 e 2017 na cidade de Sobral (CE).

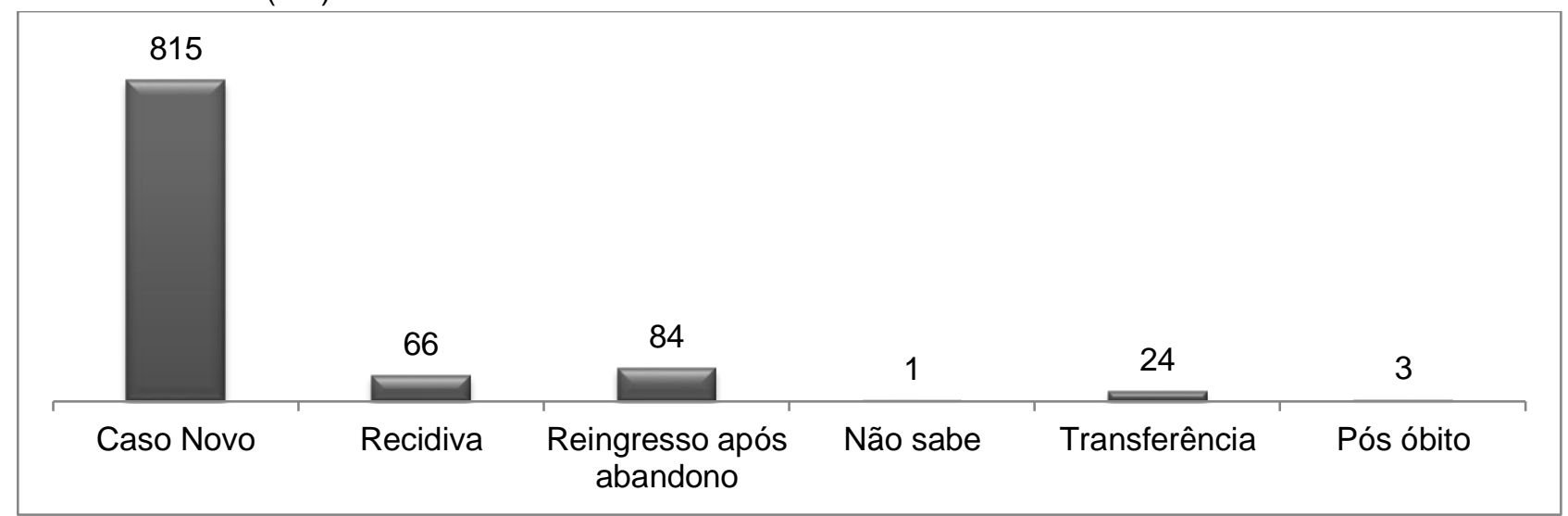

Fonte: Dados da pesquisa. Ministério da Saúde/SVS - Sistema de Informação de Agravos de Notificação SINAN Net

As notificações dos casos de tuberculose pulmonar constituíram em maior número, com $84 \%$, seguidos dos casos extrapulmonares com $13 \%$ dos eventos notificados. Em menor percentual, $2 \%$, estiveram os casos em que as apareceram as duas formas clínicas da doença.

Em relação ao método prioritário para identificação do doente bacilífero, observou-se que a maior parcela dos casos notificados $(54,0 \%)$ era positiva para o bacilo na primeira baciloscopia direta do escarro. No entanto, evidenciou-se uma alta taxa (31,5\%) de casos onde não houve realização do método (Tabela 2).

Tabela 2 - Casos de tuberculose, em relação à primeira baciloscopia de escarro, notificados entre os anos de 2013 e 2017 na cidade de Sobral (CE).

\begin{tabular}{|c|c|c|c|c|c|c|c|c|c|c|}
\hline \multirow{8}{*}{ 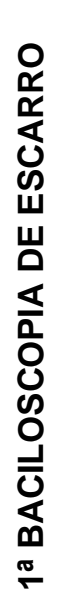 } & \multirow{2}{*}{ Ano Notificação } & \multicolumn{2}{|c|}{ Positivo } & \multicolumn{2}{|c|}{ Negativo } & \multicolumn{2}{|c|}{ Não realizado } & \multicolumn{2}{|c|}{ Não se aplica } & \multirow{2}{*}{ Total } \\
\hline & & $\mathbf{N}$ & $\%$ & $\mathbf{N}$ & $\%$ & $\mathbf{N}$ & $\%$ & $\mathbf{N}$ & $\%$ & \\
\hline & 2013 & 109 & 55,1 & 36 & 18,2 & 53 & 26,8 & - & - & 198 \\
\hline & 2014 & 108 & 49,8 & 28 & 12,9 & 80 & 36,9 & 1 & 0,5 & 217 \\
\hline & 2015 & 105 & 53,8 & 10 & 5,1 & 72 & 36,9 & 8 & 4,1 & 195 \\
\hline & 2016 & 105 & 57,7 & 20 & 11,0 & 49 & 26,9 & 8 & 4,4 & 182 \\
\hline & 2017 & 109 & 54,2 & 19 & 9,5 & 59 & 29,4 & 14 & 7,0 & 201 \\
\hline & TOTAL & 536 & 54,0 & 113 & 11,4 & 313 & 31,5 & 31 & 3,1 & 993 \\
\hline
\end{tabular}

Fonte: Dados da pesquisa. Ministério da Saúde/SVS - Sistema de Informação de Agravos de Notificação SINAN Net. 
Ainda em relação à baciloscopia de escarro, quando se contabilizaram os casos notificados de segunda baciloscopia, os dados demonstraram que $62 \%$ foram ignorados/branco e $18 \%$ nem realizaram o exame, os quais se somam $80 \%$ de casos onde não se conheceram os resultados para a segunda baciloscopia de escarro.

Para a situação de encerramento a cura constituiu maior percentual com 613 casos notificados. Entretanto, o número de abandono (77 casos) e óbito por tuberculose (49 casos) foi significativo, bem como o valor de casos ignorados/branco, sendo estes aumentados em 2017, ano no qual se observou um total de 103 notificações onde a situação de encerramento foi ignorada (Gráfico 3).

Gráfico 3 - Casos de tuberculose, conforme situação de encerramento, notificados entre os anos de 2013 e 2017 na cidade de Sobral (CE).

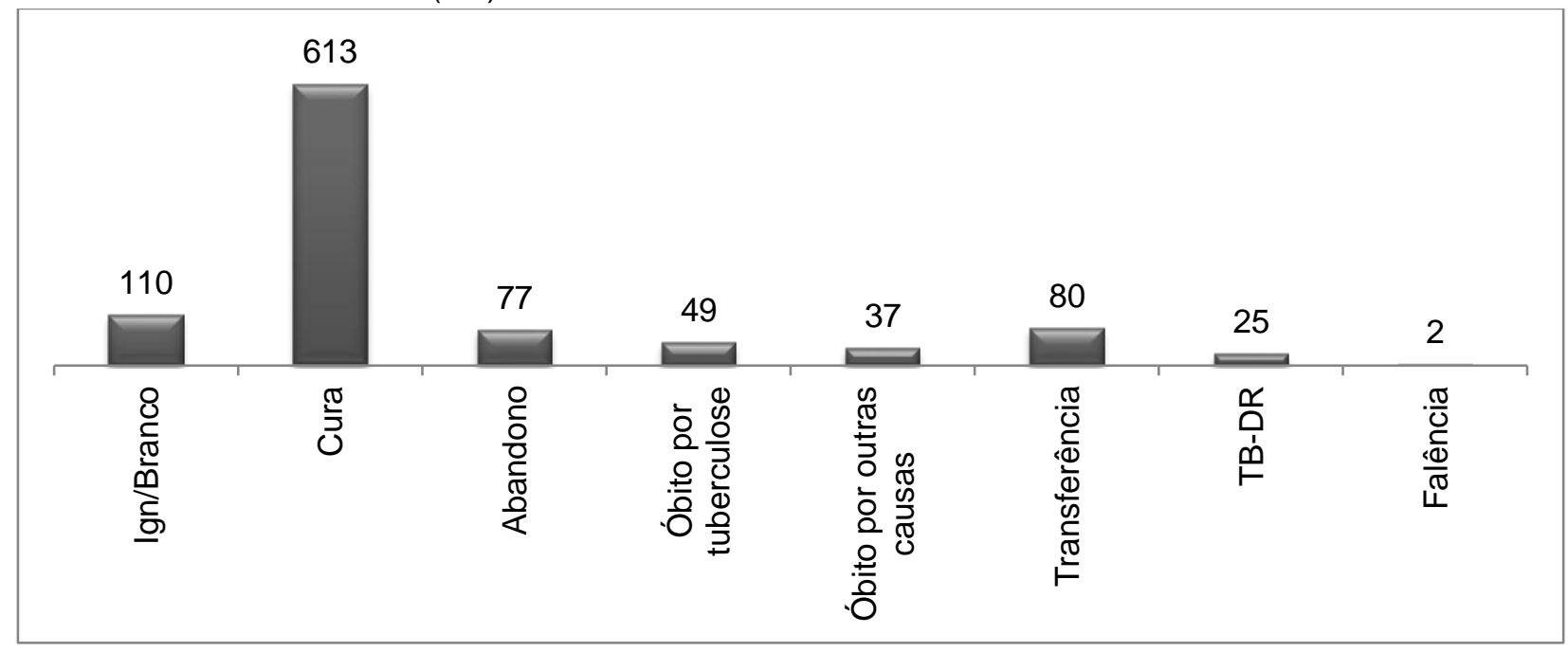

Fonte: Dados da pesquisa. Ministério da Saúde/SVS - Sistema de Informação de Agravos de Notificação - SINAN Net.

\section{DISCUSSÃO}

Diversos são os fatores que colaboram para a alta incidência da tuberculose em uma determinada região. Os dados apresentados neste artigo demonstram o cenário epidemiológico da tuberculose no município de Sobral (CE), onde nos anos de 2015 e 2016 houve um declínio em relação às notificações da tuberculose, entretanto com aumento significativo em 2017.

É importante questionar se a diminuição dos novos casos foi relacionada a uma real redução da doença no município, ou se houve uma carência na ação dos serviços de saúde em relação ao conhecimento concreto dos novos casos e consequentemente ao diagnóstico precoce e notificação dos indivíduos (FARIAS EJS, et al., 2013).

Em 2017 foi realizada uma ação pela equipe da Vigilância Epidemiológica de Sobral (CE) juntamente com equipes dos Centros de Saúde da Família (CSF) de alguns bairros da cidade, do Núcleo de Apoio a Saúde da Família (NASF) e residentes em Saúde da Família para a assistência a usuários do Centro de Referência Especializado para População em Situação de Rua de Sobral (Centro POP), onde foram concretizados atendimentos com coletas de escarro para baciloscopia, além de outros exames e orientações acerca dos sintomas, diagnóstico e tratamento da tuberculose (JÚNIOR M, 2017).

Sugere-se que tal ação tenha parcela de responsabilidade em relação ao aumento no número de notificações de tuberculose na cidade de Sobral (CE) no ano de 2017, visto que a busca ativa se torna uma ferramenta útil para a evidenciação dos casos em que o paciente não procura o serviço de saúde (CECILIO HPM, et al., 2015). 
Quanto à faixa etária de infecção por TB têm-se os adolescentes com padrão idêntico ao do adulto, entretanto crianças menores expressam a TB de maneira inespecífica e muitas vezes assintomática, o que pode acarretar em dificuldade no diagnóstico e, consequente, aumento do risco de evolução dos casos para as formas mais graves e até a morte (CANO APG, et al., 2017).

Em relação ao sexo acometido, vários estudos corroboram os resultados encontrados onde a maioria das notificações, internações e óbitos por TB ocorreram na população masculina. Tal configuração pode ser aplicada pelo fato dos homens estarem mais ausentes dos serviços de saúde e assim, demorar mais no diagnóstico e no início do tratamento. Dados do Ministério da Saúde apontaram que $66,8 \%$ dos casos de tuberculose registrados em 2014 foram entre homens (CALIARI JS e FIGUEIREDO RM, 2012; CECILIO HPM, et al., 2013; CHAVES EC, et al., 2017).

Os casos notificados, conforme tipo de entrada, classificados como pós-óbito constituem outro dado importante, visto que demonstra a qualidade dos serviços de saúde, ou seja, são aqueles dados que não foram registrados no SINAN e notificados após a morte do indivíduo. Tal dado supõe a fragilidade do serviço em detectar as pessoas com tuberculose, seja pela falha na busca ativa de casos novos e consequentes, diagnóstico tardio, seja na identificação dos contatos, bem como a qualidade da assistência prestada à população (CEARÁ, 2018). Como perspectiva de estudo seria a busca por mais informações quanto à correlação da TB com a causa do óbito desses usuários.

Ao identificar a forma clínica têm-se a tuberculose pulmonar mais prevalente e a mais relevante para a saúde pública, visto que é responsável pela conservação da transmissão da doença entre os indivíduos (FARIAS EJS, et al., 2013). Este dado coincide com o resultado encontrado nesse artigo, uma vez que o pulmão é a porta de entrada para o Mycobacterium tuberculosis, o qual pode se disseminar por contiguidade, ocasionando a forma pleural, por via linfática (forma ganglionar) ou hematogênica (pleural, ganglionar e demais formas extrapulmonares) (CHAVES EC, et al., 2017).

Os métodos fundamentais de diagnóstico consistem de avaliação clínica baseada em sintomas descritos pelo paciente e na situação epidemiológica na qual ele se encontra, bem como no diagnóstico por meio de exames laboratoriais. A realização da baciloscopia de escarro constitui estratégia primordial na busca dos casos da tuberculose na população com tosse e expectoração por mais de 2 semanas, sendo principal método diagnóstico devido a seu custo reduzido, coleta de fácil execução e rápido resultado. Entretanto, estudos observam uma sensibilidade média de 40 a $60 \%$, sendo positivo em apenas $20 \%$ dos pacientes com lesão pulmonar mínima. Outros agravantes incluem a análise, o volume da secreção e as condições de armazenamento (JACOMELLI M, et al., 2012).

Muitos são os esforços para a superação de desafios relacionados ao diagnóstico da tuberculose, principalmente no que diz respeito aos casos de infecção latente. Ressalta-se a constante busca por novas metodologias para a rápida detecção e universalização do diagnóstico, contribuindo para a identificação de casos de TB latente, sensível e resistente (BARREIRA D, 2018).

Na perspectiva do desfecho clínico dos casos, observou-se na pesquisa que, em todos os anos estudados, a situação de encerramento relacionado à cura prevaleceu sobre os outros casos, o que pode sugerir um correto manejo no tratamento e o acompanhamento dos indivíduos por parte dos profissionais, ainda que diante de dificuldades existentes nos serviços de saúde (FARIAS EJS, et al., 2013).

Por outro lado, a proporção de abandono ao tratamento de tuberculose em Sobral (CE) ainda é alta correspondendo a $11,0 \%$, resultado esse que se apresenta acima do que é preconizado pela OMS (>5\%). Além disso, é importante ressaltar o número elevado de reingresso após abandono do tratamento, o qual evidencia que ainda são necessários esforços para evitar estes casos, visto que podem progredir para agravamento da doença e possíveis fontes de contaminação para o restante da população (BRASIL, 2016a).

O número de óbitos, apesar de baixo, deve ser considerado como significativo, uma vez que a tuberculose se configura como uma doença tratável. As principais causas de óbitos por tuberculose englobam o acesso tardio e dificultoso, tanto do diagnóstico quanto do tratamento, quando os pacientes já se encontram em 
estágios avançados da doença, reduzindo a eficácia do tratamento e consequentemente as chances de cura. A coinfecção, principalmente HIV/TB é outro fator que aumenta sobremaneira os índices de mortalidade por tuberculose (PINHEIRO RS, et al., 2012; FARIAS EJS, et al., 2013).

Os limites dos resultados desta pesquisa estão pautados no uso de dados secundários do Sistema de Informação de Agravos de Notificação e pela qualidade dessas informações. Por outro lado, a utilização dos conteúdos disponíveis no sistema de informação em saúde permite o acompanhamento do agravo, contribuindo para a identificação de aspectos importantes e impulsionando a investigação de novas estratégias para o controle da doença (CECILIO HPM, et al., 2013).

\section{CONCLUSÃO}

Diversos são os fatores que colaboram para a alta incidência da TB em uma determinada região. Os dados apresentados neste trabalho demonstram que, apesar da TB ser uma doença tratável e curável, ainda é significativo o número de indivíduos acometidos por essa doença. Desta maneira, destacam-se os indicadores epidemiológicos de saúde como importante instrumento capaz de refletir a qualidade dos serviços prestados à população, servindo como apoio para a tomada de decisão em Saúde Pública. Ressalta-se que a busca por novas ferramentas e mudanças no diagnóstico e no tratamento da TB ainda são lentas, entretanto as tecnologias e drogas existentes são fundamentais no controle da doença.

\section{REFERÊNCIAS}

1. ALCOBIA CS, COSTA SAN. Tuberculose na grávida: um relato de caso. Revista Portuguesa de Medicina Geral e Familiar, 2016; 32:350-2.

2. BARREIRA D. Os desafios para a eliminação da tuberculose no Brasil. Epidemiologia e Serviços de Saúde, Brasília, 2018; 27(1):e00100009.

3. BRASIL. Ministério da Saúde. Secretaria de Vigilância em Saúde. Boletim Epidemiológico: Perspectivas brasileir as para o fim da tuberculose como problema de saúde pública. v. 47, n. 13. - 2016a.

4. Ministério da Saúde. Secretaria de Vigilância em Saúde. Departamento de Vigilância Epidemiológica. Doenças infecciosas e parasitárias: guia de bolso. 8 ed. Ver. Brasília: Ministério da Saúde, 2010, 448 p.

5. Ministério da Saúde. Secretaria de Vigilância em Saúde. Departamento de Vigilância das Doenças Transmissíveis. Rede de Teste Rápido para Tuberculose no Brasil: primeiro ano da implantação. - Brasília: Ministério da Saúde, 2015. $63 \mathrm{p}$.

6. Ministério da Saúde. Secretaria de Vigilância em Saúde. Departamento de Vigilância das Doenças Transmissíveis. Panorama da tuberculose no Brasil: a mortalidade em números [recurso eletrônico]. Brasília: Ministério da Saúde, $2016 b, 126 \mathrm{p}$.

7. Ministério da Saúde. Teste Rápido Molecular para Tuberculose (TRM-TB): nova tecnologia para o diagnóstico da tuberculose. - Brasília: Ministério da Saúde, 2016c.

8. Programa Nacional de Controle da Tuberculose. Apresentação sobre os principais indicadores da tuberculose. $2016 \mathrm{~d}$.

9. CALIARI JS, FIGUEIREDO RM. Tuberculose: perfil de doentes, fluxo de atendimento e opinião de enfermeiros. Acta Paulista de Enfermagem, 2012; 25(1):43-7.

10. CEARÁ. Coordenadoria de Promoção e Proteção à Saúde. Núcleo de Vigilância Epidemiológica. Secretaria da Saúde do Estado do Ceará. Boletim Epidemiológico: tuberculose. 2018.

11. CECILIO HPM, et al. Acesso ao diagnóstico de tuberculose sob a ótica dos profissionais de saúde. Texto \& Contexto Enfermagem, 2017; 26(3):e0230014.

12. CECILIO HPM, et al. Perfil das internações e óbitos hospitalares por tuberculose. Acta Paulista de Enfermagem, 2013; 26(3):250-5.

13. CECILIO HPM, et al. Opinião dos profissionais de saúde sobre os serviços de controle da tuberculose. Acta Paulista de Enfermagem, 2015; 28(1):19-25.

14. CHAVES EC, et al. Aspectos epidemiológicos, clínicos e evolutivos da tuberculose em idosos de um hospital universitário em Belém, Pará. Revista Brasileira de Geriatria e Gerontologia, 2017; 20(1): 47-58.

15. FARIAS EJS, et al. Análise epidemiológica dos casos de tuberculose notificados no município de Sobral - CE no período de 2007 a 2011. SANARE, 2013; 12(1):33-39.

16. JACOMELLI M, et al. Broncoscopia no diagnóstico de tuberculose pulmonar em pacientes com baciloscopia de escarro negativa. Jornal Brasileiro de Pneumologia, 2012; 38:167-73.

17. OMS. Organização Mundial de Saúde. Global tuberculosis report 2015. Geneva: OMS, 2015.

18. PINHEIRO RS, et al. Subnotificação da tuberculose no Sistema de Informação de Agravos de Notificação (SINAN): abandono primário de bacilíferos e captação de casos em outras fontes de informação usando linkage probabilístico. Caderno de Saúde Pública, 2012; 28(8):1559-1568.

19. SINAN. Sistema de Informação de Agravos de Notificação. Tuberculose no Ceará entre os anos de 2013 e 2017. 